\title{
Determining fractional Zener model parameters from low frequency DMA measurements
}

\author{
Nadine Feldmann ${ }^{1}$, Fabian Bause ${ }^{1}$, Bernd Henning ${ }^{1}$ \\ ${ }^{1}$ Measurement Engineering Group, Paderborn University, Warburger Straße 100, \\ 33098 Paderborn, Germany \\ feldmann@emt.uni-paderborn.de
}

\begin{abstract}
The present contribution addresses the problem of characterising frequency dependent mechanical material properties of linear viscoelastic materials. Therefore, the material behaviour for a wide frequency range is calculated from several DMA measurements using the time-temperaturesuperposition. These so-called master curves describe the material behaviour for a broad frequency range, which is necessary for obtaining the parameters of a rheological model. Here, the fractional Zener model is applied and parameterised to represent the viscoelastic properties of a polyamide 6 (PA6) specimen.
\end{abstract}

Key words: fractional Zener model, dynamic mechanical analysis, time temperature superposition, elastic modulus, optimisation

\section{Introduction}

In order to apply certain materials in a mechanical system, it is important to characterise its physical properties. Since the material behaviour depends among others on the frequency, for fully describing the material it is necessary to determine the properties at different frequencies. A simple and widespread method for studying the material's performance at low frequencies is the Dynamic Mechanical Analysis (DMA). Since the applied frequencies are typically below $100 \mathrm{~Hz}$, the time-temperaturesuperposition can be deployed to extend the measuring range. From several DMA measurements at different temperatures, it is possible to conclude the higher frequency behaviour. Mostly, it is reasonable to not assign a value to each frequency, but to parameterise a frequency dependent model. Thus, only a small amount of parameters is able to represent all relevant aspects. These parameters have to be determined for each material. This can be achieved by finding those parameters that are best suited for representing measured data. In this contribution, a procedure is presented using DMA measurements for parameterising a fractional Zener model for representing linear viscoelastic material behaviour.

\section{Time-dependent Viscoelastic Material Behaviour}

The mechanical behaviour of a material specimen can be described by determining the relation between the applied mechanical tension $\sigma$ and the occurring strain $\varepsilon$. An ideal elastic material is characterised by a proportional relationship (Hooke's law):

$$
\sigma=E \varepsilon
$$

A viscose material however follows a timedependent relation, e.i. the stress is proportional to the derivative of the stress with respect to time:

$$
\sigma=\eta \frac{\mathrm{d} \varepsilon}{\mathrm{d} t}
$$

Viscoelastic materials show a combination of these two behaviours. They are usually described by various models containing the elastic moduli $E$ describing elastic components and viscosities $\eta$ describing the viscous parts. [1]

The effects of linear viscoelasticity can be interpreted as retardation (creep) and relaxation mechanisms. The retardation time constant $\tau_{\varepsilon}$ describes the response time of the material, when a step in stress is applied, whereas the relaxation time constant $\tau_{\sigma}$ describes the response time of the decaying stress when an abrupt strain is applied. These time constants can be measured by quasi-static tensile creep tests. [2]

Well-known, simple models such as the KelvinVoigt- or the Maxwell-modell do often not suffice to describe the material entirely. They can either represent retardation or relaxation mechanisms, but not both. Thus more complex models such 
as the fractional Zener model described in the following are required.

\section{Fractional Zener Model}

The Zener model [3] (also known as standard linear solid model) can be interpreted as a combination of two springs (denoting the elastic moduli $E_{\mathrm{a}}$ and $E_{\mathrm{M}}$ ) and one dashpot (denoting the material coefficient of viscosity $\eta_{\mathrm{M}}$ ) arranged as shown in figure 1

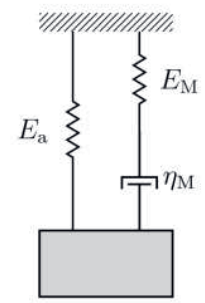

Fig. 1. Mechanical spare model for the Zener model

The mathematical description for such a system is given by the differential equation

with

$$
\sigma+\tau \dot{\sigma}=E_{\mathrm{a}} \varepsilon+\left(E_{\mathrm{M}}+E_{a}\right) \tau \dot{\varepsilon}
$$

$\dot{\sigma}=\frac{\mathrm{d} \sigma}{\mathrm{d} t}, \dot{\varepsilon}=\frac{\mathrm{d} \varepsilon}{\mathrm{d} t}$ and $\tau=\frac{\eta_{\mathrm{M}}}{E_{\mathrm{M}}}$.

This model is able to describe retardation and relaxation at the same time, but only in an exponential manner. The relaxation time $\tau_{\sigma}$ is $\tau$ itself, the retardation time $\tau_{\varepsilon}$ can be calculated to be $\frac{E_{\mathrm{M}}+E_{\mathrm{a}}}{E_{\mathrm{M}} E_{\mathrm{a}}} \eta_{\mathrm{M}}$.

A more general model is the fractional Zener model using fractional derivatives of order $\kappa$ and $\lambda(0<\kappa, \lambda \leq 1)$, that are defined as [4]:

$$
\frac{\mathrm{d}^{\kappa}}{\mathrm{d} t^{\kappa}} f(t)=\frac{1}{\Gamma(1-\kappa)} \frac{\mathrm{d}}{\mathrm{d} t} \int_{0}^{x} \frac{\dot{f}\left(t^{*}\right)}{\left(t-t^{*}\right)^{\kappa}} d t^{*}
$$

Different modifications of this fractional Zener model can be applied, however the fiveparameter model which is used here is given by the differential equation [5]

$$
\begin{aligned}
\sigma+\tau^{\lambda} \frac{\mathrm{d}^{\lambda}}{\mathrm{d} t^{\lambda}} \sigma= & E_{0} \varepsilon+E_{0} \tau^{\lambda} \frac{\mathrm{d}^{\lambda}}{\mathrm{d} t^{\lambda}} \varepsilon \\
& +\left(E_{\infty}-E_{0}\right) \tau^{\kappa} \frac{\mathrm{d}^{\kappa}}{\mathrm{d} t^{\kappa}} \varepsilon .
\end{aligned}
$$

The retardation and relaxation processes are no longer restricted to exponential behaviour, but can be described as being in between a hyperbolic and exponential one (Mittag-Leffler function).

This model is able to represent a wide variety class of linear viscoelastic materials.

\section{Harmonic Analysis of Viscoelastic Behaviour}

When transforming the time-dependent viscoelastic differential equations into the frequency domain, the behaviour can be characterised by a complex modulus $E=E^{\prime}+$ $\mathrm{j} E^{\prime \prime}$, where $E^{\prime}$ is the storage modulus and $E^{\prime \prime}$ is the loss modulus. Assuming a harmonic strain excitation $\varepsilon(t)=\varepsilon_{0} \sin \left(2 \pi f_{0} t\right)$ the harmonic stress response is phase-shifted:

$$
\begin{aligned}
\sigma(t)= & \sigma_{0} \sin \left(2 \pi f_{0} t+\delta\right) \\
= & \sigma_{0} \cos (\delta) \sin \left(2 \pi f_{0} t\right) \\
& +\sigma_{0} \sin (\delta) \cos \left(2 \pi f_{0} t\right)
\end{aligned}
$$

Thus, the stress contains one component inphase with the strain excitation and one component shifted by $90^{\circ}$. The complex, frequency-dependent modulus then is given by

$$
\begin{aligned}
E\left(f=f_{0}\right) & =\frac{\sigma(t)}{\varepsilon(t)} \\
& =\frac{\sigma_{0}}{\varepsilon_{0}} \cos (\delta)+\mathrm{j} \frac{\sigma_{0}}{\varepsilon_{0}} \sin (\delta) \\
& =E^{\prime}+\mathrm{j} E^{\prime \prime} .
\end{aligned}
$$

The modulus usually follows a characteristic function of the logarithmic scaled frequency depicted in figure 2.

For measuring this frequency-dependent viscoelastic behaviour, a Dynamic Mechanical Analysis (DMA) can be applied.

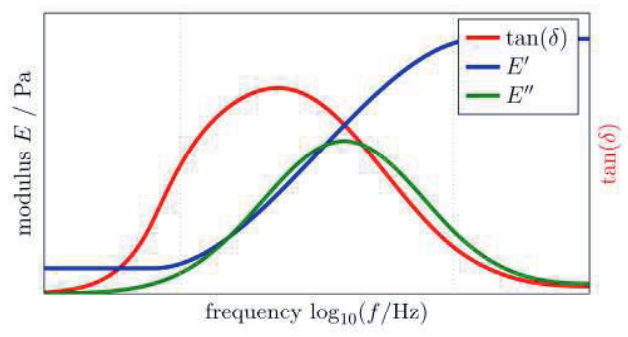

Fig. 2. Characteristic curve of the frequency dependant mechanical moduli

\section{Dynamic Mechanical Analysis (DMA)}

The Dynamic Mechanical Analysis [6] is a measurement technique for characterising the frequency-dependent material behaviour for frequencies lower than the specimens resonance frequency (typically below $100 \mathrm{~Hz}$ ).

A harmonic stress is applied with increasing frequency whereupon the resulting strain is measured. Different modes of deformation are possible, like shear, torsional or tensile stress. Another possibility is the dual cantilever bending. Here, both ends of a thin specimen are clamped and a drive shaft applies the harmonic stress on the specimens' centre. The whole set-up is positioned in a chamber with controlled temperature, which is quite important in the following. 


\section{Time Temperature Superposition}

Since the DMA measurements usually are conducted using low frequencies, but the determination of the parameters of a viscoelastic model require the material behaviour over a broad frequency range, a single DMA measurement is not well suited for this purpose. Nevertheless, the low-frequency data obtained by the DMA can be extrapolated to frequencies orders of magnitude larger. This can be achieved using the time-temperature-superposition.

The principle of time-temperature superposition describes, that a higher (lower) frequency has the same effect on the mechanical behaviour as a lower (higher) temperature. This seems plausible when picturing a material that gets stiffer, when high frequency stress is applied similar to when it is cooled down. This means that a change of temperature causes a scaling of the frequency / time axis which is equal to a frequency / time shift of the logarithmic frequency / time. [7]

Thus, the low-frequency DMA measurements can be performed at different temperatures and these can be shifted along the frequency axis to describe the higher-frequency range. From several single measurements one can create a so called master curve describing the material behaviour for several decades of frequency $/$ time as depicted in figure 3 .

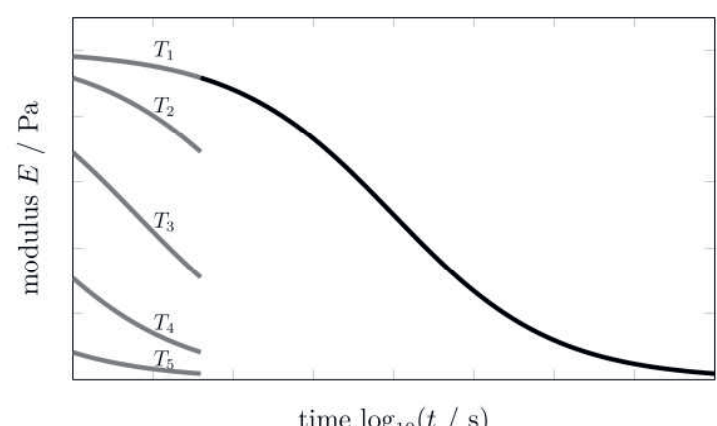

Fig. 3. Exemplary master curve generation

Mathematically, the William-Landel-Ferry equations allow the calculation of the temperature dependent frequency scaling coefficients $a_{T}(T)$ [8]. Starting from a temperature $T_{0}$ the frequency dependent measurements of another temperature $T$ have to be shifted along the logarithmic frequency for $\log _{10}\left(a_{T}(T)\right)$. A linear relation is given by

$$
-\frac{1}{\log _{10}\left(a_{T}(T)\right)}=\frac{C_{2}}{C_{1}} x+\frac{1}{C_{1}}
$$

with

$$
x=\frac{1}{T-T_{0}}
$$

and the material constants $C_{1}$ and $C_{2}$.

Since this simple relationship between frequency and temperature is not always given, the time-temperature-superposition does only apply to so called thermorheologically simple materials. Whether a material displays this simple relationship can easily be seen in a ColeCole-plot, i.e. displaying the elastic modulus in the complex plane for different temperatures. If this results in a continuous semicircular curve, the material can be regarded as thermorheologically simple. [9]

Figure 4 depicts the Cole-Cole-plot for the measured modulus of PA6. The typical curve for themorheologically simple material is recognisable, although certain deviations can be observed for lower temperatures.

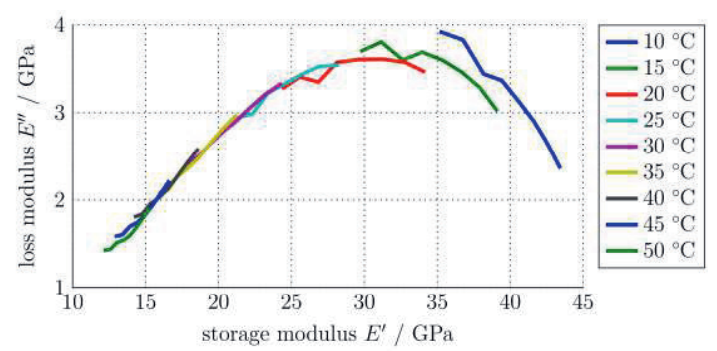

Fig. 4. Cole-Cole-plot for PA6 measurements

\section{Measurement Set-up and Master Curve Generation}

The DMA measurements have been carried out in the dual cantilever mode using a PA6 specimen of size $20 \mathrm{~mm} \times 5.8 \mathrm{~mm} \times 2.02 \mathrm{~mm}$ in a temperature range from $10^{\circ} \mathrm{C}$ to $50^{\circ} \mathrm{C}$ and a frequency range from $0.1 \mathrm{~Hz}$ to $21.5 \mathrm{~Hz}$. The shifting along the frequency axis is conducted such that a continuous master curve results (see figure 5).
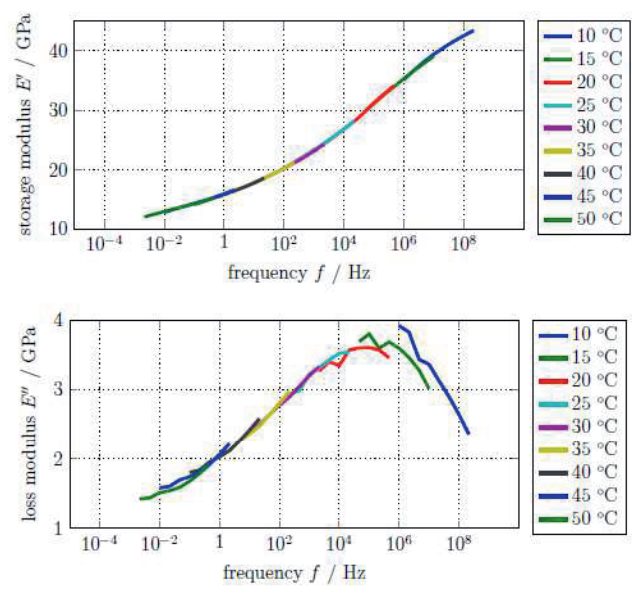

Fig. 5. Generated master curves for PA6

The deviations for low temperature measurements that were already observed in the 
Cole-Cole-plot can mainly be seen in the loss modulus.

Since some calibration problems concerning the phase angle $\delta$ occurred during the measurement, some corrections had to be made in order to obtain physically valid measurement data.

\section{Determining the Fractional Zener Model Parameters from DMA Measurements}

When a master curve from DMA-measurements is found, one can determine the parameters for a fractional Zener model from this curve. Therefore a description of the time-dependent differential equations is Fourier transformed and the complex modulus can be calculated [5]:

$$
E(\mathrm{j} f)=E_{0}+E_{0}(d-1) \frac{(\mathrm{j} 2 \pi f \tau)^{\kappa}}{1+(\mathrm{j} 2 \pi f \tau)^{\lambda}}
$$

with

$$
d=\frac{E_{\infty}}{E_{0}}
$$

For a physical model, all parameters have to be non-negative and for the orders of the fractional derivatives $\kappa$ and $\lambda$, the relation $\kappa>\lambda$ is given. For polymers $\Delta=\kappa-\lambda<0.06$ does typically apply.

In the following, the model parameters $E_{0}, E_{\infty}, \tau, \kappa$ and $\Delta$ are fitted to the master curve via an optimisation process. Since most optimisation algorithms need initial values, these are approximated directly from the master curves using the methods from table 1.

Tab. 1: Initial value estimation for fractional Zener model parameters

\begin{tabular}{|c|l|}
\hline Parameter & Determination method \\
\hline$E_{0}$ & Storage modulus at $f=0$ \\
\hline$E_{\infty}$ & Storage modulus at $f \rightarrow \infty$ \\
\hline $2 \pi \tau$ & $\begin{array}{l}\text { Reciprocal of the frequency at } \\
\text { maximal storage modulus }\end{array}$ \\
\hline$\kappa$ & $\begin{array}{l}\text { slope of the logarithm of the loss } \\
\text { modulus before its maximum }\end{array}$ \\
\hline$\Delta$ & 0.03 for polymers \\
\hline
\end{tabular}

The optimisation bounds for the parameters are set such that a physically meaningful result is obtained. For the optimisation, a LevenbergMarquardt-algorithm is applied. The results are listed in table 2.
Tab. 2: Initial and optimised values for the fractional Zener model parameters

\begin{tabular}{|c|c|c|}
\hline Parameter & Initial value & Optimised value \\
\hline$E_{0}$ & $12 \mathrm{GPa}$ & $10.35 \mathrm{GPa}$ \\
\hline$E_{\infty}$ & $4.166 \mathrm{GPa}$ & $5.251 \mathrm{GPa}$ \\
\hline $2 \pi \tau$ & $21.277 \mu \mathrm{s}$ & $5.254 \mu \mathrm{s}$ \\
\hline$\kappa$ & 0.2342 & 0.1595 \\
\hline$\Delta$ & 0.03 & 0.000223 \\
\hline
\end{tabular}

The comparison between the measured and generated master curves and the optimised model is depicted in figure 5 . One sees immediately that they are in good agreement except for the high-frequency part of the loss modulus, which already showed some deviations in the master curve generation.
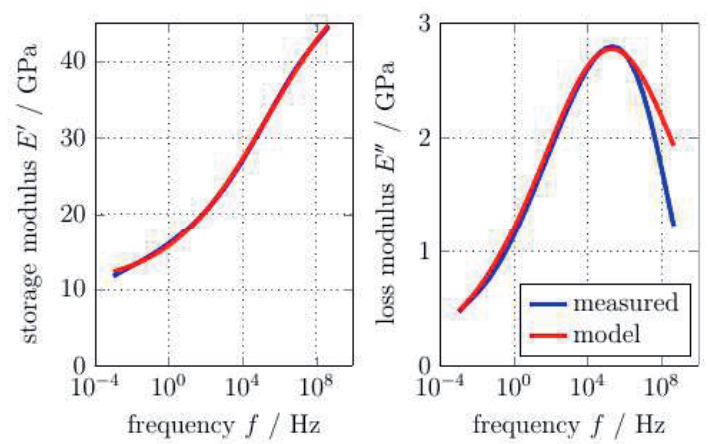

Fig. 5. Generated master curves for PA6

Thus, the mechanical behaviour of the PA6 specimen is given by the fractional Zener model and it's parametrisation for a large frequency range.

\section{Conclusions}

In summary, it was possible to use the timetemperature-superposition in order to obtain the elastic properties of PA6 for a broad range of frequencies from several low-frequency measurements. Furthermore, the measured data could be brought into agreement with a fiveparameter fractional Zener model by calculating fitting parameters. Thus, the viscoelastic behaviour can be described not only for the measured frequency range, but - using the analytic model - for arbitrary frequencies as long as the fractional Zener model is applicable.

Another restriction for the applicability of the presented measurement technique is the thermorheological simplicity. The DMA measurements carried out with a polyether ether ketone (PEEK) specimen could not be shifted to form a continuous master curve and thus, no material parameters could be identified. 
A comparison with alternative measurement techniques for determining mechanical properties of polymers that intrinsically obtain higher frequency results can be found in [10].

\section{Acknowledgements}

We would like to thank Christopher Schulte and the department of Coating Materials and Polymers for supporting us with the realisation of the DMA measurements. Furthermore, the authors would like to acknowledge the financial support of the German Research Foundation (DFG) for the research project HE 2897/6-2.

\section{References}

[1] I.M. Ward, D.W. Hadley, An introduction to the mechanical properties of solid polymers, Wiley (2011)

[2] International Standard ISO 899:2003: Plastics — Determination of creep behaviour

[3] C. Zener, Elasticity and Anelasticity of Metals, The University of Chicago Press (1948)

[4] M. Caputo, Linear Models of Dissipation whose Q is almost Frequency Independent - II,

Geophysical Journal international 13.5, 529-539

(1967); doi: 10.1111/j.1365-246X.1967.tb02303.x

[5] T. Pritz, Five-parameter fractional derivative model for polymeric damping materials, Journal of Sound and Vibration 265.5, 935-952 (2003); doi: 10.1016/S0022-460X(02)01530-4

[6] International Standard ISO 67211:2012, Plastics - Determination of dynamic mechanical properties, (2012)

[7] R.S. Lakes, Viscoelastic measurement techniques, Review of Scientific Instruments 75.4, 797-810 (2004); doi: 10.1063/1.1651639

[8] M.L. Williams, R.F. Landel, J.D. Ferry, The Temperature Dependence of Relaxation Mechanisms in Amorphous Polymers and Other Glass-forming Liquids, Journal of the American Chemical Society 77.14, 3701-3707 (1955); doi: 10.1021/ja01616a088

[9] D.I.G. Jones, Handbook of Viscoelastic Vibration Damping, Wiley (2001)

[10] J. Rautenberg, F. Bause, B. Henning, Utilizing guided acoustic waves to measure dispersive material properties of polymers, Sensor Conference 2015 (2015); doi: 10.5162/sensor2015/A6.1 